Article

\title{
Dynamics of a Protein Interaction Network Associated to the Aggregation of polyQ-Expanded Ataxin-1
}

\author{
Aimilia-Christina Vagiona ${ }^{1}$, Miguel A. Andrade-Navarro ${ }^{2}$ (D), Fotis Psomopoulos ${ }^{3,4}(\mathbb{C}$ \\ and Spyros Petrakis ${ }^{3, *}$ (iD \\ 1 Department of Biology, Aristotle University of Thessaloniki, 54124 Thessaloniki, Greece; \\ emilyvagiona@gmail.com \\ 2 Faculty of Biology, Johannes Gutenberg University, Biozentrum I, Hans-Dieter-Hüsch-Weg 15, \\ 55128 Mainz, Germany; andrade@uni-mainz.de \\ 3 Institute of Applied Biosciences/Centre for Research and Technology Hellas, 57001 Thessaloniki, Greece; \\ fpsom@certh.gr \\ 4 Department of Molecular Medicine and Surgery, Karolinska Institutet, 17177 Stockholm, Sweden \\ * Correspondence: spetrak@certh.gr; Tel.: +30-2311-257-525
}

Received: 20 August 2020; Accepted: 23 September 2020; Published: 25 September 2020

\begin{abstract}
Background: Several experimental models of polyglutamine (polyQ) diseases have been previously developed that are useful for studying disease progression in the primarily affected central nervous system. However, there is a missing link between cellular and animal models that would indicate the molecular defects occurring in neurons and are responsible for the disease phenotype in vivo. Methods: Here, we used a computational approach to identify dysregulated pathways shared by an in vitro and an in vivo model of ATXN1(Q82) protein aggregation, the mutant protein that causes the neurodegenerative polyQ disease spinocerebellar ataxia type-1 (SCA1). Results: A set of common dysregulated pathways were identified, which were utilized to construct cerebellum-specific protein-protein interaction (PPI) networks at various time-points of protein aggregation. Analysis of a SCA1 network indicated important nodes which regulate its function and might represent potential pharmacological targets. Furthermore, a set of drugs interacting with these nodes and predicted to enter the blood-brain barrier (BBB) was identified. Conclusions: Our study points to molecular mechanisms of SCA1 linked from both cellular and animal models and suggests drugs that could be tested to determine whether they affect the aggregation of pathogenic ATXN1 and SCA1 disease progression.
\end{abstract}

Keywords: ataxin-1; polyQ; pathway; network; drugs; blood-brain-barrier

\section{Introduction}

The polyglutamine (polyQ) disease spinocerebellar ataxia type-1 (SCA1) is a lethal, progressive, autosomal dominant neurodegenerative disorder caused by a CAG trinucleotide expansion in the ataxin-1 (ATXN1) gene [1]. This mutation produces a longer polyQ tract in the pathogenic protein which gradually misfolds into an abnormal conformation and forms protein inclusions within the nuclei of neurons [2]. Cerebellar neurons that coordinate movement are particularly sensitive to ATXN1 aggregation; their gradual dysfunction and loss are responsible for the characteristic symptoms of SCA1, including loss of coordination and ataxia [3,4].

Formation of polyQ inclusions is the main feature of SCA1 pathology; however, other factors also affect the progression of the disease. Several studies have shown that ATXN1 interacts with transcription regulators, RNA splicing factors and other nuclear receptors [5-8], suggesting that alterations of these interactions by the polyQ expansion in the mutant protein might drive cerebellar 
pathology [9]. To develop treatments for this disease, it might beneficial to employ approaches from network medicine, which studies disease in terms of the pathological effects of altered protein interaction networks [10-12]. Neurodegenerative diseases characterized by protein aggregates, which spread in the nervous system [13], have been studied from this point of view [14,15].

In order to gain insights into the pathogenesis of SCA1, various experimental models of the disease have been previously generated, including induced pluripotent stem cells [16] and transgenic mice expressing human ATXN1, with an expanded polyQ tract $[17,18]$. SCA1 B05 mice are widely used for modeling this disease in vivo, since they develop Purkinje cell degeneration, indicating that a mouse model can be established simply by introducing CAG repeat expansions in a wild-type protein. Purkinje cells gradually accumulate nuclear inclusions which increase with age; approximately $90 \%$ of these neurons contain polyQ inclusions by week 12 . In parallel, mice at increasing ages, (week 5 , 12 and 28) show symptoms corresponding to mild, moderate and severe ataxia, respectively [17]. Recent studies showed that ATXN1 expression levels are regulated by miR760 binding to a conserved region in its 5' untranslated region [19], while the ATXN1-Capicua (CIC) protein complex is the main driver of pathology in the cerebellum through a gain-of-function mechanism [9]. Furthermore, analysis of the cerebellar transcriptome of SCA1 B05 mice indicated gene networks whose expression profiles correlate with disease progression in the cerebellum [20]. These networks were also dysregulated in a different SCA1 in vivo model [18] and differ considerably to other regions of the brain, including the medulla [21].

We previously developed an inducible cellular model of ATXN1(Q82) protein aggregation in human mesenchymal stem cells (Tet-On YFP-ATXN1(Q82) MSCs), in which the pathogenic protein gradually forms insoluble intranuclear inclusions. These inclusions cause oxidative and nucleolar stress, affect the assembly of the ribosome and eventually lead to cell necrosis. Furthermore, a number of transcriptional changes were identified which correlate with the gradual aggregation of polyQ-expanded ATXN1 in human SCA1 cerebellum, as well. These include dysregulation of the protein synthesis machinery and pathways involved in focal adhesion or oxidative phosphorylation [20]. This inducible model offers the possibility to identify specific molecular changes in vitro which may contribute to the selective neuropathological phenotype in vivo.

Here we attempted to identify common molecular changes in Tet-On YFP-ATXN1(Q82) MSCs and SCA1 B05 mice. Proteins participating in dysregulated pathways at different time-points of polyQ aggregation were used for the construction of perturbed cerebellum-specific protein-protein interaction (PPI) networks. Our analysis indicates important nodes of a SCA1 PPI network affected by the gradual aggregation of pathogenic ATXN1. Pharmacological targeting of these proteins may modify polyQ aggregation and SCA1 disease progression.

\section{Materials and Methods}

\subsection{Datasets of SCA1 B05 Transgenic Mice and Tet-On YFP-ATXN1(Q82) MSCs}

RNA-seq datasets from the cerebellum of three age groups ( $n=3$ animals per group) of SCA1 B05 transgenic mice (week 5, week 12 and week 28) and age-matched control FVB mice were retrieved from the literature [21]. The GEO accession number of these RNA-seq data is GSE75778. The DIOPT (DRSC Integrative Ortholog Prediction Tool) tool, which integrates ortholog predictions from 11 commonly used orthology tools [22], was used to map human orthologs of murine genes. Only human genes with a high rank score were selected.

RNA-seq datasets from Tet-On YFP-ATXN1(Q82) MSCs at three different time-points of polyQ-expanded protein aggregation (Day 2, Day 5 and D10) and a control time-point (Day 0) ( $n=3$ samples per time point) were selected. These data are publicly available [20]. The final list contained only genes that are specifically dysregulated by the expression of pathogenic ATXN1. 


\subsection{Differential Gene Expression Analysis}

Gene expression levels were measured in "fragments per kilobase of exon model per million mapped reads" (FPKM) values [23]. A mean FPKM value from the experimental triplicates was calculated for each gene. For differential gene expression analysis, a fold change (FC) for each gene was calculated using the following equation and FC data were $\log _{2}$ normalized.

$$
\mathrm{FC}=[\mathrm{FPKM} \mathrm{g}(\mathrm{SCA} 1)] /[\mathrm{FPKM} \mathrm{g}(\mathrm{control})]
$$

Genes from SCA1 B05 transgenic mice (week 5-week 28) were compared to FVB control mice at the same age; genes from Tet-On YFP-ATXN1(Q82) MSCs at Day 2-Day 10 were compared to cells at Day 0. A t-test was applied to compare FPKM levels between a group and its respective control. Genes with $\mid \log _{2} \mathrm{FCl}>0.5$ and $p$-value $<0.05$ were considered as DEGs (differentially expressed genes) and used for further analysis. Differential expression analysis was performed using $\mathrm{R}$ version 3.6.1 programming software (RStudio Team 2016).

\subsection{Pathway Enrichment Analysis}

DEGs were used for pathway enrichment analysis in the online tool Enrichr (version 2.1) [24]. Dysregulated pathways in SCA1 B05 transgenic mice and Tet-On YFP-ATXN1(Q82) MSCs were identified using the Kyoto Encyclopedia of Genes and Genomes (KEGG) database and were ranked by $p$-value, as calculated by the EnrichR platform. KEGG pathways from mice and cells were compared and common pathways at each time-point with a $p$-value $<0.05$ were selected.

\subsection{Construction of PPI Networks}

Protein components of the common dysregulated pathways were used for the construction of PPI networks using the STRING database [25] in Cytoscape (version 3.7.2) [26]. Only genes expressed in the nervous system (score of 4.8 using the relevant tissue filter) [27] and high confidence protein interactions (score of 0.950 or above) were utilized. Unconnected nodes were deleted. Statistical significance for the networks was measured using the Motif Discovery plug-in of Cytoscape (version 0.0.3). A z-score for 4-node motifs was calculated for each network after comparison with 1000 random networks. The topological properties of the networks were calculated using the Network Analyzer plugin of Cytoscape [28] and were visualized using the GraphPad Prism software.

\subsection{Selection of Genes Analysis}

The online tool Génie was used to select mouse genes associated to the biomedical research in protein aggregates in the brain [29]. Basically, this tool uses a user-defined query to PubMed to retrieve a non-specific set of records that is used to define a score for the words enriched in the set. In a second search, PubMed records are evaluated for their content in the enriched words and genes from a species linked to those records are ranked. Génie also allows gene selection via orthologs. Here, we used Génie with default thresholds, using the query "protein aggregation brain" to rank mouse genes, using human orthologs to extend the literature.

\subsection{Network Analysis}

NetworkAnalyzer was used to compute the topological centralities of PPI networks. Genes were extracted based on three criteria: (a) degree centrality (DC), (b) betweenness centrality (BC) and (c) closeness centrality (CC).

DC of each node, i, was defined as:

$$
\mathrm{D}(\mathrm{i})=\Sigma \mathrm{jm}(\mathrm{i}, \mathrm{j})
$$

where $m(i, j)=1$ if there is a link from node $i$ to node $j$. 
$\mathrm{BC}$ referring to the frequency of node $\mathrm{i}$ appearing at nodes $\mathrm{j}$ and $\mathrm{k}$ was calculated by the equation:

$$
\mathrm{B}(\mathrm{i})=\Sigma \mathrm{a}, \mathrm{bgjik} / \mathrm{gjk}
$$

where $i \neq j \neq k$, gjk is the number of the shortest pathways between nodes $j$ and $k$, gjik is the number of the shortest pathways containing $i$.

$\mathrm{CC}$ of a node $\mathrm{i}$ in a graph was calculated by the equation:

$$
\mathrm{C}(\mathrm{i})=\Sigma \mathrm{j} \mathrm{d}(\mathrm{i}, \mathrm{j})
$$

where $\mathrm{i} \neq \mathrm{j}$, dij is the shortest pathway between nodes $\mathrm{i}$ and $\mathrm{j}$.

Important nodes/proteins were identified based on topological networks analysis.

\subsection{Drug-Protein Interaction Network}

A drug-protein interaction network was constructed for selected proteins (coefficient score of 0.5) using the Cytoscape plugin CyTargetLinker [30]. Drug-protein interactions were retrieved from the DrugBank database [31]. The online tool Blood Brain Barrier Predictor [32] was utilized to assess whether selected drugs can penetrate the blood-brain barrier (BBB).

\section{Results}

\subsection{Identification of Dysregulated Pathways in Tet-On YFP-ATXN1(Q82) MSCs and SCA1 B05 Transgenic Mice}

First, we attempted to identify dysregulated pathways in two different experimental models of polyQ-expanded human ATXN1, which show a consistent dysregulation in gene expression due to the aggregation of the mutant protein. The computational workflow used here is summarized in Figure 1. Gene datasets were selected from an in vitro cell model and an in vivo mouse model of protein aggregation, namely, Tet-On YFP-ATXN1(Q82) MSCs and SCA1 B05 transgenic mice, respectively. Several of the selected DEGs, at least in the in vitro cell model, were independently validated by quantitative MS [22]. Importantly, both models expressed full length human ATXN1 harboring the same pathogenic polyglutamine length. In order to compare the two datasets, murine genes from the SCA1 B05 transgenic mice were first converted into their human orthologs. Then, the change in their expression levels was calculated at three different time-points of protein aggregation [week 5 (W5) — early, week 12 (W12) — middle and week 28 (W28)-late] compared to the respective age-matched control group (FVB mice). Genes with a mean $\left|\log _{2} \mathrm{FC}\right|>0.5$ and $<0.05$, indicating a consistent expression among triplicates, were considered as DEGs and were used for further analysis. Similarly, using the same criteria, DEGs were selected from Tet-On YFP-ATXN1(Q82) MSCs at three different time-points of protein aggregation [Day 2 (D2)—early, Day 5 (D5)—middle and Day 10 (D10)—late] (Supplementary Table S1). These cells gradually accumulate insoluble polyQ-inclusions compared to control [Day 0 (D0)] Tet-On TFP-ATXN1(Q82) MSCs. The majority of selected DEGs in both Tet-On YFP-ATXN1(Q82) MSCs and SCA1 B05 transgenic mice were downregulated, as previously described [20,21]. The two datasets shared 143 genes which showed a significant agreement in the direction of fold change with 38 genes upregulated in at least one time-point in both models ( $p$-value 0.011): these 38 upregulated genes were functionally enriched for genes involved in extracellular matrix organization (Benjamini corrected $p$-value $1.7 \times 10^{-6}$ ), but not the 33 genes with downregulation in both models or the remaining ones. Furthermore, these 143 common genes are enriched in binding sites for the ETV4 transcription factor (Supplementary Table S2) whose activity is regulated by CIC, the main driver of pathogenesis in SCA1 [33]. 


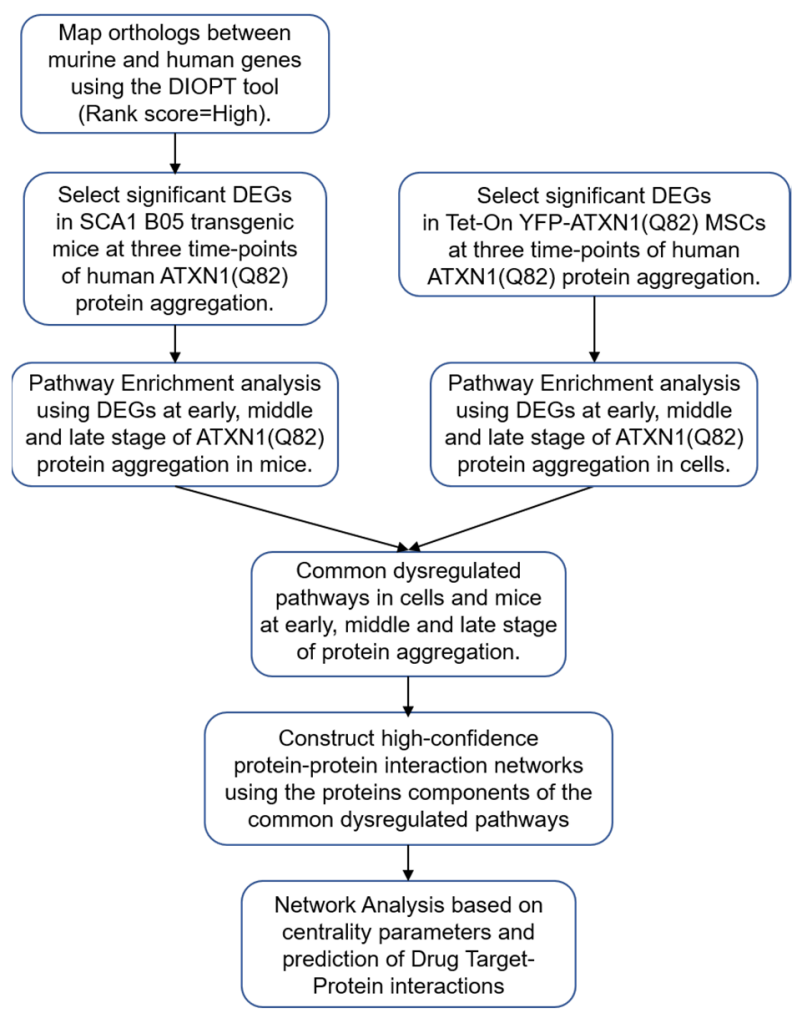

Figure 1. Data workflow for the construction of PPI networks associated to protein aggregation in Tet-On YFP-ATXN1(Q82) MSCs and SCA1 B05 transgenic mice.

In order to identify dysfunctional pathways associated with gradual protein aggregation, DEGs in each dataset and time-point were used for enrichment analysis using the KEGG database. Only pathways with a $p$-value $<0.05$ were considered as significantly dysregulated at each time-point (Figure 1). Using the DEGs at D2 cells $(n=687)$ and W5 mice $(n=357)$, this analysis identified 28 and 18 pathways, respectively, that were dysregulated at an early time-point of polyQ protein aggregation in the two experimental models. Similarly, DEGs at D5 cells $(n=789)$ and mice at W12 $(n=1204)$ were categorized in 36 and 58 pathways, respectively. Finally, DEGs $(n=801$ at D10 cells and $n=1063$ at week 28 mice) indicated 32 pathways in each experimental model that were associated with a late stage of polyQ-expanded ATXN1 protein aggregation (Supplementary Table S3).

We hypothesized that the gradual aggregation of ATXN1(Q82) into insoluble inclusions in MSCs may have similar molecular characteristics to progressive ataxia observed in B05 mice with aging. Therefore, we matched the selected time-points from cells and mice (D2 MSCs to W5 mice, D5 MSCs to W12 mice and D10 MSCs to W28 mice) and asked which dysregulated pathways were common in both protein aggregation models at each time-point. Three pathways, protein digestion and absorption, ECM-receptor interaction and PI3K-Akt signaling pathway were identified at an early stage of protein aggregation. Interestingly, these pathways were dysregulated at all further time-points in both models. At a middle stage, five more pathways were identified, including ribosome biogenesis, Alzheimer's and Parkinson's disease. Two of them, Rap1 signaling pathway and focal adhesion were dysregulated also at a later time-point. Furthermore, regulation of actin cytoskeleton and AGE-RAGE signaling pathway specifically featured the later stage, when polyQ-expanded ATXN1 forms terminal inclusions (Table 1) 
Table 1. Common dysregulated pathways in cells and mice at matched time-points associated with protein aggregation.

\begin{tabular}{|c|c|c|c|c|}
\hline \multirow[b]{2}{*}{ Enrichment Term } & \multicolumn{2}{|c|}{ Cells } & \multicolumn{2}{|c|}{ Mice } \\
\hline & Overlap & $p$-Value & Overlap & $p$-Value \\
\hline \multicolumn{5}{|c|}{ A. Day 2 (D2) Cells vs. Week 5 (W5) Mice } \\
\hline Protein digestion and absorption & $8 / 90$ & 0.012 & $6 / 90$ & 0.005 \\
\hline ECM-receptor interaction & $14 / 82$ & $>0.001$ & $5 / 82$ & 0.016 \\
\hline PI3K-Akt signaling pathway & $26 / 341$ & $>0.001$ & $12 / 341$ & 0.002 \\
\hline \multicolumn{5}{|c|}{ B. Day 5 (D5) Cells vs. Week 12 (W12) Mice } \\
\hline Ribosome & $38 / 137$ & $>0.001$ & $17 / 137$ & 0.003 \\
\hline ECM-receptor interaction & $18 / 82$ & $>0.001$ & $12 / 82$ & 0.003 \\
\hline Focal adhesion & $24 / 202$ & $>0.001$ & $21 / 202$ & 0.009 \\
\hline PI3K-Akt signaling pathway & $29 / 341$ & $>0.001$ & $30 / 341$ & 0.022 \\
\hline Protein digestion and absorption & $12 / 90$ & 0.001 & $11 / 90$ & 0.018 \\
\hline Alzheimer's disease & $15 / 168$ & 0.002 & $18 / 168$ & 0.011 \\
\hline Rap1 signaling pathway & $16 / 211$ & 0.001 & $21 / 211$ & 0.015 \\
\hline Parkinson's disease & $11 / 142$ & 0.024 & $14 / 142$ & 0.045 \\
\hline \multicolumn{5}{|c|}{ C. Day 10 (D10) Cells vs. Week 28 (W28) Mice } \\
\hline AGE-RAGE signaling pathway & 9/101 & 0.019 & $11 / 101$ & 0.012 \\
\hline ECM-receptor interaction & $13 / 82$ & $>0.001$ & $9 / 82$ & 0.03 \\
\hline Focal adhesion & $21 / 202$ & $>0.001$ & $17 / 202$ & 0.042 \\
\hline PI3K-Akt signaling pathway & $26 / 341$ & 0.001 & $27 / 341$ & 0.026 \\
\hline Protein digestion and absorption & $8 / 90$ & 0.027 & $9 / 90$ & 0.05 \\
\hline Rap1 signaling pathway & $16 / 211$ & 0.01 & $22 / 211$ & 0.002 \\
\hline Regulation of actin cytoskeleton & $18 / 214$ & 0.002 & $22 / 214$ & 0.002 \\
\hline
\end{tabular}

The Table shows the common dysregulated pathways at (A) early, (B) middle and (C) late stage of protein aggregation in Tet-On YFP-ATXN1(Q82) MSCs and SCA1 B05 transgenic mice and the overlap with the components of the pathways. The analysis was performed using the Kyoto Encyclopedia of Genes and Genomes (KEGG) database.

\subsection{Perturbed PPI Networks in SCA1 Models}

Following previous strategies that studied the mechanisms of neurodegenerative disease from a network medicine perspective [14], we generated PPI networks associated with the gradual aggregation of pathogenic ATXN1 using as input the proteins that participate in the common dysregulated pathways of the SCA1 models. Three perturbed PPI networks were generated at each time-point (D2 cells/W5 mice: $z$-score $=-0.981$, D5 cells/W12 mice: $z$-score $=-0.990$ and D10 cells/W28 mice: $z$-score $=-0.976$ ), which included only high-confidence PPIs of proteins produced in the nervous system (Supplementary Figure S1). The largest network was observed at a middle stage of protein aggregation. The protein nodes of these networks and the pathways in which they participate are shown in (Supplementary Table S4). These networks were significantly enriched in genes related to protein aggregates in the brain, particularly the middle stage network (see Methods for details) (Supplementary Figure S2). We note that aggregation-related genes in the early network were also present in the middle and late networks, but aggregation-related genes present in the middle network were not in the late network.

Then, PPIs of these networks were combined into a large network, which is perturbed by the gradual aggregation of pathogenic ATXN1 (Figure 2A). The SCA1 PPI network (z-score $=-0.977$ ) contains discrete clusters and subnetworks associated with various stages of protein aggregation, perturbed either at all time-points ( $n=50$, yellow color), or specifically at a middle or late time-point ( $n=87$ green or $n=38$ magenta color, respectively) (Figure 2B and Supplementary Table S5). Specifically, the yellow cluster of ATP1 proteins is involved in sodium ion transport and is perturbed at all time-points of protein aggregation (Supplementary Table S6A). The green cluster participates in ATP synthesis as most of these proteins are components of the mitochondrial respiratory chain (Supplementary Table S6B). Finally, ribosomal proteins and proteins participating in G-protein signaling pathway 
form highly interconnected modules within the SCA1 PPI network (Supplementary Table S6C,D, respectively).

A.

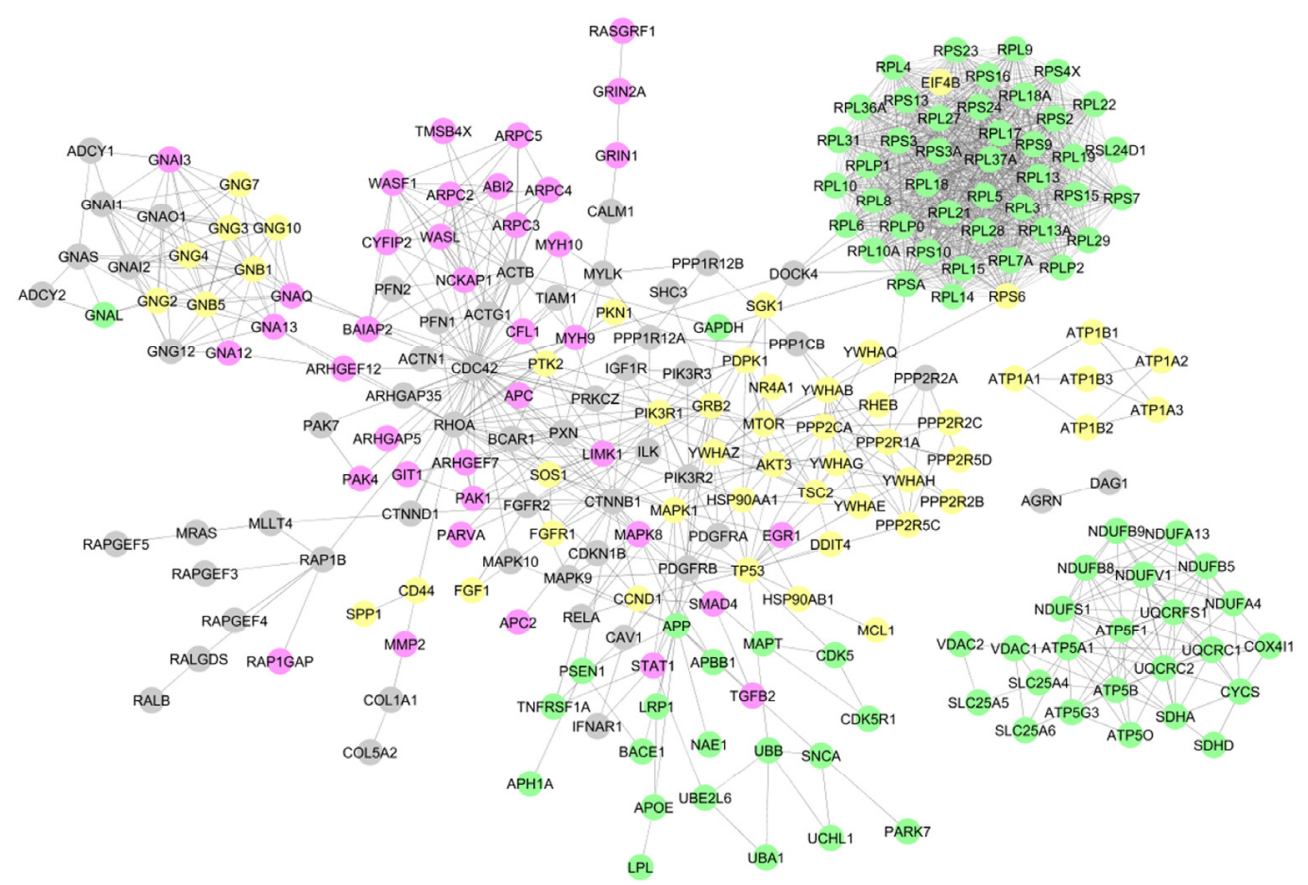

B.

\begin{tabular}{|c|c|c|c|}
\hline & \multicolumn{3}{|c|}{$\begin{array}{l}\text { SCA1 network nodes per time point } \\
\text { of protein aggregation }\end{array}$} \\
\hline & Early & Middle & Late \\
\hline \multicolumn{4}{|c|}{$n=15$} \\
\hline \multicolumn{4}{|c|}{$n=87$} \\
\hline \multicolumn{4}{|c|}{$n=40$} \\
\hline \multicolumn{4}{|c|}{$n=38$} \\
\hline$=50$ & & & \\
\hline
\end{tabular}

Figure 2. SCA1 disease PPI network. (A) The network contains 230 nodes (proteins) and 1432 edges (interactions). Green and magenta nodes are dysregulated at middle and late stage of protein aggregation, respectively. Yellow color indicates nodes that are dysregulated at all time-points. The network was constructed using the STRING database in Cytoscape. (B) Gannt chart indicating the different groups of nodes in the SCA1 disease network. Colors indicate the same groups as in (A).

\subsection{Analysis of SCA1 PPI Networks}

Next, we analyzed the connectivity and functionality of the SCA1 PPI network by calculating the degree (DC), betweenness (BC) and closeness (CC) centralities of its components at all time-points (early, middle and late stage of protein aggregation). We identified 11 proteins (early: PPP2CA, TP53, MTOR and PIK3R, middle: RPS6, RPL15 and RPS3, late: CDC42, RHOA, PIK3R1 and CTNNB1) with the highest DC values, namely, the number of connections of each node. These proteins were forming important hubs within the individual networks (Supplementary Figure S3).

However, the importance of a node in a biological network does not depend only on its number of neighbors [34] but it may increase as a node participates in communication paths and controls the flow of information (BC) [35] or it has a central role in the network being closer to all the other nodes (CC). Thus, we identified important nodes with the highest BC values (early: GNB1, GNB5 and MTOR, middle: CD44, PIK3R2 and YWHAH, late: CDC42, RHOA and GNB1) (Supplementary Figure S4). 
On the other hand, ATP1 protein subunits (ATP1A1, ATP1A2, ATP1A3, ATP1B1, ATP1B2 and ATP1B3), which formed a discrete cluster within the network, had the highest $C C$ values at all three stages of protein aggregation (Supplementary Figure S5). The numerical values of DC, BC and CC for all nodes of the SCA1 PPI network are show in Supplementary Table S7.

Centrality changes may indicate whether a node gains or loses its importance within a network [36]. Therefore, we attempted to link time-dependent changes in the centralities of the highly ranked nodes with the dynamic changes of the SCA1 network, which trigger various cellular mechanisms as the disease progresses. In total, 21 proteins were selected based on their DC, BC and CC values. The changes in their centralities during polyQ protein aggregation are visualized in Figure 3. These heatmaps indicate that CDC42 and RHOA, which are involved in the regulation of the cell cycle and the formation of stress fibers [37], gradually increase their DC and BC (Figure 3A,B). In contrast, GNB1 and GNB5, which are involved in the transduction of various transmembrane signals in cells, including neurons [38], show a significant decrease in their BC and CC (Figure 3B,C). Furthermore, the CC of CD44, a cell-surface receptor involved in cell adhesion and regulating the functionality of dendritic spines [39] is dramatically decreased (Figure 3C). In contrast, ATP1 subunits do not show any obvious change in their centralities at all time-points (Figure 3).

A.

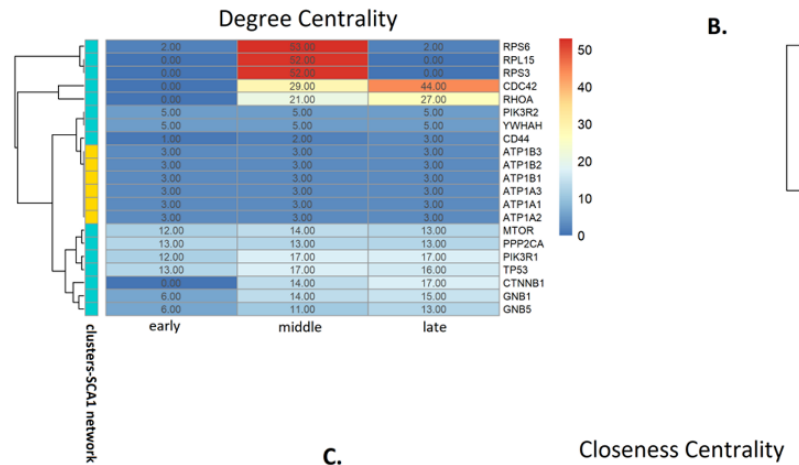

c.

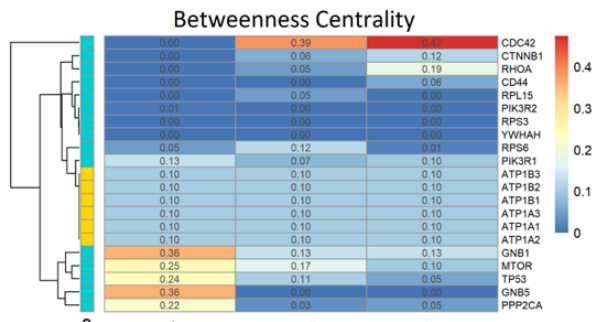

early

middle

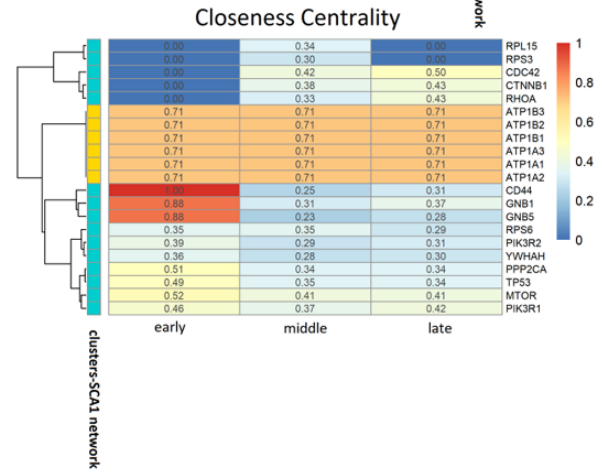

Figure 3. Centrality heatmaps of 21 nodes of the SCA1 disease network with the higher DC, BC and CC values per time-point. (A). Degree, (B). betweenness and (C). closeness centralities at early, middle or late time-point of protein aggregation. (B). Color range from blue (low) to red (high) indicates scaled centrality values. Chromatic code on the left side of each heatmap indicates in which cluster of the SCA1 network each node belongs.

\subsection{Drug-Protein Interaction Network in SCA1}

Several lines of evidence indicate that nodes of a network with increased DC and BC values represent potential drug targets $[40,41]$. In contrast, reduced centrality may characterize nodes that gradually lose their function and need to be stimulated. A number of SCA1 network components (GNB1, PPP2CA, MTOR, TP53, CDC42, RHOA and ATP1A1) were further prioritized as potential targets for pharmacological intervention. These proteins were considered important regulatory nodes of the SCA1 network, based on the gradual change of their centralities (Figure 3). GNB1, PPP2CA, MTOR and TP53 encode proteins with decreased BC and CC centralities, while DC, BC and CC centralities 
of CDC42 and RHOA increased over time. Additionally, ATP1A, a representative component of the discrete cluster of the network was also selected, based on its constantly high CC value. A drug-protein interaction network was constructed, depicting the interactions between 7 target-proteins and 32 drugs which are which are either FDA-approved or under evaluation in clinical trials (Supplementary Figure S6). The majority of them $(n=16)$ bind to ATP1A1 subunit.

We then sought to investigate whether these drugs may enter the brain, which is the organ mainly affected in SCA1. Drugs with an 8/8 positive score in the algorithm/fingerprint combinations were predicted to cross the BBB (Supplementary Table S8). The drugs and corresponding protein targets include: Vitamin E for PP2AC, PhiKan 083 and AZD 3355 for TP53, FARNESYL for GNB1, and Bretylium and Ciclopirox for ATP1A1 (Table 2). These drugs may be used for pharmacological targeting of proteins of the central nervous system which regulate the function of the SCA1 network that is disturbed during polyQ-expanded protein aggregation.

Table 2. Drugs that interact with components of the SCA1 protein network and are predicted to penetrate the blood brain barrier.

\begin{tabular}{|c|c|c|c|c|}
\hline Target & Drug & Algorithm & Fingerprint & BBB Permeability Prediction \\
\hline \multirow{8}{*}{ PPP2AC } & \multirow{8}{*}{ Vitamin E } & \multirow{4}{*}{ ADABoost } & MACCS & $\mathrm{BBB}+$ \\
\hline & & & Openbabel & $\mathrm{BBB}+$ \\
\hline & & & Molprint & $\mathrm{BBB}+$ \\
\hline & & & PubChem & $\mathrm{BBB}+$ \\
\hline & & \multirow{4}{*}{ SVM } & MACCS & $\mathrm{BBB}+$ \\
\hline & & & Openbabel & $\mathrm{BBB}+$ \\
\hline & & & Molprint & $\mathrm{BBB}+$ \\
\hline & & & PubChem & $\mathrm{BBB}+$ \\
\hline \multirow{16}{*}{ TP53 } & \multirow{8}{*}{ PhiKan 083} & \multirow{4}{*}{ ADABoost } & MACCS & $\mathrm{BBB}+$ \\
\hline & & & Openbabel & $\mathrm{BBB}+$ \\
\hline & & & Molprint & $\mathrm{BBB}+$ \\
\hline & & & PubChem & $\mathrm{BBB}+$ \\
\hline & & \multirow{4}{*}{ SVM } & MACCS & $\mathrm{BBB}+$ \\
\hline & & & Openbabel & $\mathrm{BBB}+$ \\
\hline & & & Molprint & $\mathrm{BBB}+$ \\
\hline & & & PubChem & $\mathrm{BBB}+$ \\
\hline & \multirow{8}{*}{ AZD 3355} & \multirow{4}{*}{ ADABoost } & MACCS & $\mathrm{BBB}+$ \\
\hline & & & Openbabel & $\mathrm{BBB}+$ \\
\hline & & & Molprint & $\mathrm{BBB}+$ \\
\hline & & & PubChem & $\mathrm{BBB}+$ \\
\hline & & \multirow{4}{*}{ SVM } & MACCS & $\mathrm{BBB}+$ \\
\hline & & & Openbabel & $\mathrm{BBB}+$ \\
\hline & & & Molprint & $\mathrm{BBB}+$ \\
\hline & & & PubChem & $\mathrm{BBB}+$ \\
\hline \multirow{8}{*}{ GNB1 } & \multirow{8}{*}{ FARNESYL } & \multirow{4}{*}{ ADABoost } & MACCS & $\mathrm{BBB}+$ \\
\hline & & & Openbabel & $\mathrm{BBB}+$ \\
\hline & & & Molprint & $\mathrm{BBB}+$ \\
\hline & & & PubChem & $\mathrm{BBB}+$ \\
\hline & & \multirow{4}{*}{ SVM } & MACCS & $\mathrm{BBB}+$ \\
\hline & & & Openbabel & $\mathrm{BBB}+$ \\
\hline & & & Molprint & $\mathrm{BBB}+$ \\
\hline & & & PubChem & $\mathrm{BBB}+$ \\
\hline
\end{tabular}


Table 2. Cont.

\begin{tabular}{|c|c|c|c|c|}
\hline Target & Drug & Algorithm & Fingerprint & BBB Permeability Prediction \\
\hline \multirow{16}{*}{ ATP1A1 } & \multirow{8}{*}{ Bretylium } & \multirow{4}{*}{ ADABoost } & MACCS & $\mathrm{BBB}+$ \\
\hline & & & Openbabel & $\mathrm{BBB}+$ \\
\hline & & & Molprint & $\mathrm{BBB}+$ \\
\hline & & & PubChem & $\mathrm{BBB}+$ \\
\hline & & \multirow{4}{*}{ SVM } & MACCS & $\mathrm{BBB}+$ \\
\hline & & & Openbabel & $\mathrm{BBB}+$ \\
\hline & & & Molprint & $\mathrm{BBB}+$ \\
\hline & & & PubChem & $\mathrm{BBB}+$ \\
\hline & \multirow{8}{*}{ Ciclopirox } & \multirow{4}{*}{ ADABoost } & MACCS & $\mathrm{BBB}+$ \\
\hline & & & Openbabel & $\mathrm{BBB}+$ \\
\hline & & & Molprint & $\mathrm{BBB}+$ \\
\hline & & & PubChem & $\mathrm{BBB}+$ \\
\hline & & \multirow{4}{*}{ SVM } & MACCS & $\mathrm{BBB}+$ \\
\hline & & & Openbabel & $\mathrm{BBB}+$ \\
\hline & & & Molprint & $\mathrm{BBB}+$ \\
\hline & & & PubChem & $\mathrm{BBB}+$ \\
\hline
\end{tabular}

Table shows the score for each algorithm/fingerprint pairing of drugs which interact with selected nodes of the SCA1 network. Drugs with an 8/8 positive score were predicted to enter BBB.

\section{Discussion}

\subsection{Dysregulated Pathways Associated to polyQ-Expanded ATXN1 Aggregation}

Even though the disease-causing mutation in SCA1 has been previously identified, the pathogenic effects of polyQ-expanded ATXN1 are still under investigation. Our analysis indicates convergent dysregulated mechanisms in vitro and in vivo which are associated to polyQ protein aggregation in a time-dependent manner. Interestingly, our analysis indicates core pathways that are dysregulated since the beginning of protein aggregation and several signaling pathways that specifically feature the late-stage of the disease, which is characterized by irreversible disease-causing defects.

A statistically significant set of upregulated genes in both models is involved in extracellular matrix organization, one of the core dysregulated pathways which might be critical for polyQ-induced neurodegeneration. ATXN1 plays a critical role in ECM remodeling during development, affecting lung alveolarization. This suggests that polyQ-induced tissue abnormalities are not specifically restricted in the brain, but might also be present elsewhere in the body [33]. Furthermore, an increasing number of studies suggest the active involvement of ECM in neurodegeneration. ECM alterations, including the co-deposition of ECM components, may result in loss of protective perineuronal nets, increased neuronal cell death and synaptic deficiencies (reviewed in [42]).

Interestingly, late-stage polyQ aggregation affects the function of several signaling pathways (e.g., regulation of actin cytoskeleton and AGE-RAGE signaling pathway), suggesting that their dysregulation may be related to the selective loss of neuronal subtypes. This may be an indirect effect of the mutant protein or an alteration of the interaction between the polyQ-expanded protein with components/regulators of these signaling pathways, as shown for huntingtin, the polyQ-expanded protein which causes Huntington's disease (HD) [43].

\subsection{Network Analysis Indicates Critical Protein Nodes for SCA1 Pathogenesis}

Neurodegeneration is a complex procedure involving the parallel dysregulation of several biological processes. These are accompanied by quantitative changes in protein interactions which affect the molecular architecture of biological networks inside a cell [44]. To date, a variety of techniques have been developed allowing the analysis of PPI networks [45] and can be used to study network biology of proteins involved in neurodegeneration, including ATXN1. The SCA1 network presented 
here is cerebellum-specific and contains network motifs, i.e., small connected sub-network patterns, at a higher frequency compared to random networks [46]. Furthermore, it is highly enriched in proteins involved in protein aggregation, validating its relevance for SCA1.

Importantly, we identified dynamic changes in the SCA1 network which are induced by the gradual aggregation of mutant ATXN1. Our analysis indicates the constant dysregulation of protein complexes involved in ion transport, accompanied by the perturbation of machineries involved in protein synthesis and oxidative phosphorylation at a middle time-point and neuronal signal transduction at a late time-point. Furthermore, the middle-stage SCA1 PPI network has the largest size, suggesting that this is a critical time-point and defects caused by polyQ aggregation beyond this stage might be irreversible. It is for this middle stage that we found the largest number of genes associated to protein aggregation in the brain.

Can we modulate disease progression by targeting critical nodes of the SCA1 network? Previous studies have shown that important nodes and potential drug targets have high degree and betweenness centralities [40,41] and their deletion is related to lethality [47]. To this end, we first ranked influential nodes of the SCA1 network based on centrality measures, as previously described [48]. Our approach indicates proteins that gradually have increased significance in the perturbed network potentially contributing to SCA1 progression and proteins that lose their significance and role in the cerebellum during mutant ATXN1 aggregation.

The proteins we selected participate in various critical processes in the nervous system and most of them are involved in the pathogenesis of HD, which is also characterized by the accumulation of protein inclusions. Rho GTPases, including CDC42 and RHOA with increased centralities in the SCA1 network, regulate neuronal cell degeneration pathways [49] and several members of these signaling pathways interact with huntingtin protein [50]. ATP1A1 with a constantly high CC value is a genetic modifier of motor deficits in HD mice [51]. Similarly, nodes with decreased centralities modulate huntingtin levels (GNB1, [52]), mediate cellular dysfunction in HD (TP53, [53]) or are involved in the translation of CAG repeat expansion mRNAs (PP2CA, [54]). Taken together, these data provide insights for selective neurodegenerative processes and suggest potential drug targets. Potentially, inhibition of nodes with increased centralities or stimulation of nodes with decreased centralities of the SCA1 network may affect the aggregation of pathogenic ATXN1.

At the moment, there is no available therapy for polyQ diseases, even though Rho/ROCK and MTOR inhibitors [55,56] or compounds entering the BBB [57] are suggested to suppress the aggregation of mutant proteins and may delay disease progression. Our approach indicates potential drug targets which are involved in the pathogenesis of HD and might be also relevant for SCA1. With regards to the computational analysis, we showed that identifying the structure and underlying motifs of the network requires several steps, most of them utilizing independently executed tools. In this context, and although efficient and reproducible, the current form of the analysis is not easily scalable, given the level of user interaction necessary to run the entire process. This evident limitation can be alleviated by ensuring interoperability between the different tools, as well as automating the execution of the pipeline. This has been partially achieved through the developed code, but full automation is planned for future iterations of the software. In conclusion, here, we propose a set of drugs that target important nodes of a cerebellum-specific SCA1 network and can also enter the BBB. These drugs could be further tested to determine whether they affect the aggregation of pathogenic ATXN1 in the brain.

Supplementary Materials: The following are available online at http://www.mdpi.com/2073-4425/11/10/1129/s1, Figure S1. PPI networks at three time-points associated with protein aggregation. The protein components of the common dysregulated pathways in Tet-On YFP-ATXN1(Q82) MSCs and SCA1 B05 transgenic mice were used for the construction of PPI networks using the STRING database. (A) The PPI network at D2/W5 consists of 65 nodes and 131 edges. (B) The PPI network at D5/W12 contains 177 nodes and 1241 edges. (C) The PPI network at D10/W28consists of 144 nodes and 409 edges. Figure S2. The Venn diagram indicates the overlap between 100 genes selected as related to research on protein aggregates in the brain (see Methods for details) and the early, middle and late networks. These overlaps are significant (hypergeometric test). Figure S3. PPI networks at (A) early, (B) middle and (C) late stage of protein aggregation. Red nodes represent proteins-hubs with the 3 higher DC values. Figure S4. PPI networks at (A) early, (B) middle and (C) late stage of protein aggregation. Red nodes 
represent proteins with the 3 higher BC values. Figure S5. PPI networks at (A) early (B) middle, and (C) late stage of protein aggregation. Red nodes represent proteins-hubs with the 3 higher CC values. Figure S6. Drug-protein interaction network. Magenta nodes represent drugs that bind to important nodes of the SCA1 network. Red arrows indicate approved drugs and blue arrows experimental drugs. Table S1. Gene expression in SCA1 B05 transgenic mice and Tet-On YFP-ATXN1(Q82) MSCs. Table indicates the expression of each gene at early (D2/W5), middle (D5/W12) and late stage (D10/W28) of protein aggregation compared to its relative control in cells and mice. Only genes with a mean $|\log 2 \mathrm{FC}|>0.5$ and $\mathrm{p}$-value $<0.05$ in at least one time-point were selected for downstream analysis. Table S2. Enrichment and promoter analysis of common dysregulated genes in SCA1 B05 transgenic mice and Tet-On YFP-ATXN1(Q82) MSCs. Pathway enrichment analysis was performed using the Reactome database. The Transfac and Jaspar databases were used for promoter analysis. Table S3. Dysregulated pathways at each time-point of protein aggregation in SCA1 B05 transgenic mice and Tet-On YFP-ATXN1(Q82) MSCs. The table also shows the dysregulated genes assigned to each pathway. Only pathways with $p$-value $<0.05$ were selected. Table S4. Genes used for the construction of SCA1 PPI networks. Log2FC values for gene expression in each time-point and experimental model are shown. The table also shows the pathways in which each gene participates. Table S5. Genes dysregulated at discrete time-points of protein aggregation. Table S6. Discrete clusters and highly interconnected modules in the SCA1 disease network. The table shows GO BP enrichment analysis for the genes of each discrete cluster/module Table S7. Centrality values (DC, BC and CC) of the SCA1 network components at each time-point of protein aggregation. Genes with the higher centrality values are depicted in Figure 3 . Table S8. Drugs that interact with components of the SCA1 protein network. Table also shows whether identified drugs are predicted to enter BBB.

Author Contributions: Conceptualization, F.P., S.P.; Methodology, A.-C.V., F.P., S.P.; Formal Analysis, A.-C.V.; M.A.A.-N., F.P., S.P.; Data Curation, A.-C.V., M.A.A.-N.; Visualization, A.-C.V., M.A.A.-N., F.P., S.P.; Supervision, F.P., S.P.; Writing-Original Draft, A.-C.V., S.P.; Writing-Review and Editing, M.A.A.-N., F.P. Equal contribution: F.P. and S.P. All authors have read and agreed to the published version of the manuscript.

Funding: This work was supported by the RDA Europe Ambassador programme, European Union's Horizon 2020 (H2020) research and innovation programme via the RDA EU 4.0 project (ref. GA no. 777388). Additionally, this work was funded by ELIXIR, the research infrastructure for life-science data. SP was supported by the Hellenic Foundation for Research and Innovation (HFRI) and the General Secretariat for Research and Technology (GSRT) [grant agreement No. 122]. The funders had no role in study design, data collection and analysis, decision to publish, or preparation of the manuscript.

Acknowledgments: The authors wish to thank Stefanos Sgardelis for his critical suggestions on the experimental procedure. The code developed in this paper is available online: https://github.com/avagiona/a-vag.

Conflicts of Interest: The authors declare no conflict of interest.

\section{References}

1. Banfi, S.; Chung, M.Y.; Kwiatkowski, T.J., Jr.; Ranum, L.P.; McCall, A.E.; Chinault, A.C.; Orr, H.T.; Zoghbi, H.Y. Mapping and cloning of the critical region for the spinocerebellar ataxia type 1 gene (SCA1) in a yeast artificial chromosome contig spanning 1.2 Mb. Genomics 1993, 18, 627-635. [CrossRef]

2. Orr, H.T. Cell biology of spinocerebellar ataxia. J. Cell Biol. 2012, 197, 167-177. [CrossRef] [PubMed]

3. Klockgether, T. Update on degenerative ataxias. Curr. Opin. Neurol. 2011, 24, 339-345. [CrossRef] [PubMed]

4. Koeppen, A.H. The pathogenesis of spinocerebellar ataxia. Cerebellum 2005, 4, 62-73. [CrossRef]

5. Lam, Y.C.; Bowman, A.B.; Jafar-Nejad, P.; Lim, J.; Richman, R.; Fryer, J.D.; Hyun, E.D.; Duvick, L.A.; Orr, H.T.; Botas, J.; et al. ATAXIN-1 interacts with the repressor Capicua in its native complex to cause SCA1 neuropathology. Cell 2006, 127, 1335-1347. [CrossRef]

6. Lim, J.; Crespo-Barreto, J.; Jafar-Nejad, P.; Bowman, A.B.; Richman, R.; Hill, D.E.; Orr, H.T.; Zoghbi, H.Y. Opposing effects of polyglutamine expansion on native protein complexes contribute to SCA1. Nature 2008, 452, 713-718. [CrossRef]

7. Serra, H.G.; Duvick, L.; Zu, T.; Carlson, K.; Stevens, S.; Jorgensen, N.; Lysholm, A.; Burright, E.; Zoghbi, H.Y.; Clark, H.B.; et al. RORalpha-mediated Purkinje cell development determines disease severity in adult SCA1 mice. Cell 2006, 127, 697-708. [CrossRef]

8. Tsuda, H.; Jafar-Nejad, H.; Patel, A.J.; Sun, Y.; Chen, H.K.; Rose, M.F.; Venken, K.J.; Botas, J.; Orr, H.T.; Bellen, H.J.; et al. The AXH domain of Ataxin-1 mediates neurodegeneration through its interaction with Gfi-1/Senseless proteins. Cell 2005, 122, 633-644. [CrossRef]

9. Rousseaux, M.W.C.; Tschumperlin, T.; Lu, H.C.; Lackey, E.P.; Bondar, V.V.; Wan, Y.W.; Tan, Q.; Adamski, C.J.; Friedrich, J.; Twaroski, K.; et al. ATXN1-CIC Complex Is the Primary Driver of Cerebellar Pathology in Spinocerebellar Ataxia Type 1 through a Gain-of-Function Mechanism. Neuron 2018, 97, 1235-1243. [CrossRef] 
10. Yeger-Lotem, E.; Sharan, R. Human protein interaction networks across tissues and diseases. Front. Genet. 2015, 6, 257. [CrossRef]

11. Taylor, I.W.; Wrana, J.L. Protein interaction networks in medicine and disease. Proteomics 2012, 12, $1706-1716$. [CrossRef] [PubMed]

12. Petrakis, S.; Andrade-Navarro, M.A. Editorial: Protein Interaction Networks in Health and Disease. Front. Genet. 2016, 7, 111. [CrossRef] [PubMed]

13. Jucker, M.; Walker, L.C. Propagation and spread of pathogenic protein assemblies in neurodegenerative diseases. Nat. Neurosci. 2018, 21, 1341-1349. [CrossRef] [PubMed]

14. Morimoto, R.I. Proteotoxic stress and inducible chaperone networks in neurodegenerative disease and aging. Genes Dev. 2008, 22, 1427-1438. [CrossRef]

15. Labbadia, J.; Morimoto, R.I. The biology of proteostasis in aging and disease. Annu. Rev. Biochem. 2015, 84, 435-464. [CrossRef]

16. Buijsen, R.A.M.; Gardiner, S.L.; Bouma, M.J.; van der Graaf, L.M.; Boogaard, M.W.; Pepers, B.A.; Eussen, B.; de Klein, A.; Freund, C.; van Roon-Mom, W.M.C. Generation of 3 spinocerebellar ataxia type 1 (SCA1) patient-derived induced pluripotent stem cell lines LUMCi002-A, B, and C and 2 unaffected sibling control induced pluripotent stem cell lines LUMCi003-A and B. Stem Cell Res. 2018, 29, 125-128. [CrossRef]

17. Burright, E.N.; Clark, H.B.; Servadio, A.; Matilla, T.; Feddersen, R.M.; Yunis, W.S.; Duvick, L.A.; Zoghbi, H.Y.; Orr, H.T. SCA1 transgenic mice: A model for neurodegeneration caused by an expanded CAG trinucleotide repeat. Cell 1995, 82, 937-948. [CrossRef]

18. Watase, K.; Weeber, E.J.; Xu, B.; Antalffy, B.; Yuva-Paylor, L.; Hashimoto, K.; Kano, M.; Atkinson, R.; Sun, Y.; Armstrong, D.L.; et al. A long CAG repeat in the mouse Sca1 locus replicates SCA1 features and reveals the impact of protein solubility on selective neurodegeneration. Neuron 2002, 34, 905-919. [CrossRef]

19. Nitschke, L.; Tewari, A.; Coffin, S.L.; Xhako, E.; Pang, K.; Gennarino, V.A.; Johnson, J.L.; Blanco, F.A.; Liu, Z.; Zoghbi, H.Y. miR760 regulates ATXN1 levels via interaction with its 5' untranslated region. Genes Dev. 2020, 34, 1147-1160. [CrossRef]

20. Laidou, S.; Alanis-Lobato, G.; Pribyl, J.; Rasko, T.; Tichy, B.; Mikulasek, K.; Tsagiopoulou, M.; Oppelt, J.; Kastrinaki, G.; Lefaki, M.; et al. Nuclear inclusions of pathogenic ataxin-1 induce oxidative stress and perturb the protein synthesis machinery. Redox Biol. 2020, 32, 101458. [CrossRef]

21. Ingram, M.; Wozniak, E.A.L.; Duvick, L.; Yang, R.; Bergmann, P.; Carson, R.; O'Callaghan, B.; Zoghbi, H.Y.; Henzler, C.; Orr, H.T. Cerebellar Transcriptome Profiles of ATXN1 Transgenic Mice Reveal SCA1 Disease Progression and Protection Pathways. Neuron 2016, 89, 1194-1207. [CrossRef] [PubMed]

22. Hu, Y.; Flockhart, I.; Vinayagam, A.; Bergwitz, C.; Berger, B.; Perrimon, N.; Mohr, S.E. An integrative approach to ortholog prediction for disease-focused and other functional studies. BMC Bioinform. 2011, 12, 357. [CrossRef] [PubMed]

23. Trapnell, C.; Williams, B.A.; Pertea, G.; Mortazavi, A.; Kwan, G.; van Baren, M.J.; Salzberg, S.L.; Wold, B.J.; Pachter, L. Transcript assembly and quantification by RNA-Seq reveals unannotated transcripts and isoform switching during cell differentiation. Nat. Biotechnol. 2010, 28, 511-515. [CrossRef] [PubMed]

24. Kuleshov, M.V.; Jones, M.R.; Rouillard, A.D.; Fernandez, N.F.; Duan, Q.; Wang, Z.; Koplev, S.; Jenkins, S.L.; Jagodnik, K.M.; Lachmann, A.; et al. Enrichr: A comprehensive gene set enrichment analysis web server 2016 update. Nucleic Acids Res. 2016, 44, W90-W97. [CrossRef] [PubMed]

25. Szklarczyk, D.; Gable, A.L.; Lyon, D.; Junge, A.; Wyder, S.; Huerta-Cepas, J.; Simonovic, M.; Doncheva, N.T.; Morris, J.H.; Bork, P.; et al. STRING v11: Protein-protein association networks with increased coverage, supporting functional discovery in genome-wide experimental datasets. Nucleic Acids Res. 2019, 47, D607-D613. [CrossRef]

26. Shannon, P.; Markiel, A.; Ozier, O.; Baliga, N.S.; Wang, J.T.; Ramage, D.; Amin, N.; Schwikowski, B.; Ideker, T. Cytoscape: A software environment for integrated models of biomolecular interaction networks. Genome Res. 2003, 13, 2498-2504. [CrossRef]

27. Santos, A.; Tsafou, K.; Stolte, C.; Pletscher-Frankild, S.; O’Donoghue, S.I.; Jensen, L.J. Comprehensive comparison of large-scale tissue expression datasets. PeerJ 2015, 3, e1054. [CrossRef]

28. Assenov, Y.; Ramirez, F.; Schelhorn, S.E.; Lengauer, T.; Albrecht, M. Computing topological parameters of biological networks. Bioinformatics 2008, 24, 282-284. [CrossRef]

29. Fontaine, J.F.; Priller, F.; Barbosa-Silva, A.; Andrade-Navarro, M.A. Genie: Literature-based gene prioritization at multi genomic scale. Nucleic Acids Res. 2011, 39, W455-W461. [CrossRef] 
30. Kutmon, M.; Kelder, T.; Mandaviya, P.; Evelo, C.T.; Coort, S.L. CyTargetLinker: A cytoscape app to integrate regulatory interactions in network analysis. PLoS ONE 2013, 8, e82160. [CrossRef]

31. Law, V.; Knox, C.; Djoumbou, Y.; Jewison, T.; Guo, A.C.; Liu, Y.; Maciejewski, A.; Arndt, D.; Wilson, M.; Neveu, V.; et al. DrugBank 4.0: Shedding new light on drug metabolism. Nucleic Acids Res. 2014, 42, D1091-D1097. [CrossRef] [PubMed]

32. Liu, H.; Wang, L.; Lv, M.; Pei, R.; Li, P.; Pei, Z.; Wang, Y.; Su, W.; Xie, X.Q. AlzPlatform: An Alzheimer's disease domain-specific chemogenomics knowledgebase for polypharmacology and target identification research. J. Chem. Inf. Model. 2014, 54, 1050-1060. [CrossRef] [PubMed]

33. Lee, Y.; Fryer, J.D.; Kang, H.; Crespo-Barreto, J.; Bowman, A.B.; Gao, Y.; Kahle, J.J.; Hong, J.S.; Kheradmand, F.; Orr, H.T.; et al. ATXN1 protein family and CIC regulate extracellular matrix remodeling and lung alveolarization. Dev. Cell 2011, 21, 746-757. [CrossRef]

34. Ozgur, A.; Vu, T.; Erkan, G.; Radev, D.R. Identifying gene-disease associations using centrality on a literature mined gene-interaction network. Bioinformatics 2008, 24, i277-i285. [CrossRef] [PubMed]

35. Yu, H.; Kim, P.M.; Sprecher, E.; Trifonov, V.; Gerstein, M. The importance of bottlenecks in protein networks: Correlation with gene essentiality and expression dynamics. PLoS Comput. Biol. 2007, 3, e59. [CrossRef]

36. Koschutzki, D.; Schreiber, F. Centrality analysis methods for biological networks and their application to gene regulatory networks. Gene Regul. Syst. Biol. 2008, 2, 193-201. [CrossRef] [PubMed]

37. Nobes, C.D.; Hall, A. Rho, rac, and cdc42 GTPases regulate the assembly of multimolecular focal complexes associated with actin stress fibers, lamellipodia, and filopodia. Cell 1995, 81, 53-62. [CrossRef]

38. Huang, Y.; Thathiah, A. Regulation of neuronal communication by G protein-coupled receptors. FEBS Lett. 2015, 589, 1607-1619. [CrossRef]

39. Roszkowska, M.; Skupien, A.; Wojtowicz, T.; Konopka, A.; Gorlewicz, A.; Kisiel, M.; Bekisz, M.; Ruszczycki, B.; Dolezyczek, H.; Rejmak, E.; et al. CD44: A novel synaptic cell adhesion molecule regulating structural and functional plasticity of dendritic spines. Mol. Biol. Cell 2016, 27, 4055-4066. [CrossRef]

40. Kotlyar, M.; Fortney, K.; Jurisica, I. Network-based characterization of drug-regulated genes, drug targets, and toxicity. Methods 2012, 57, 499-507. [CrossRef]

41. Tong, Z.; Zhou, Y.; Wang, J. Identifying potential drug targets in hepatocellular carcinoma based on network analysis and one-class support vector machine. Sci. Rep. 2019, 9, 10442. [CrossRef] [PubMed]

42. Bonneh-Barkay, D.; Wiley, C.A. Brain extracellular matrix in neurodegeneration. Brain Pathol. 2009, 19, 573-585. [CrossRef] [PubMed]

43. Anzilotti, S.; Giampa, C.; Laurenti, D.; Perrone, L.; Bernardi, G.; Melone, M.A.; Fusco, F.R. Immunohistochemical localization of receptor for advanced glycation end (RAGE) products in the R6/2 mouse model of Huntington's disease. Brain Res. Bull. 2012, 87,350-358. [CrossRef]

44. Hosp, F.; Vossfeldt, H.; Heinig, M.; Vasiljevic, D.; Arumughan, A.; Wyler, E.; Landthaler, M.; Hubner, N.; Wanker, E.E.; Lannfelt, L.; et al. Quantitative interaction proteomics of neurodegenerative disease proteins. Cell Rep. 2015, 11, 1134-1146. [CrossRef] [PubMed]

45. Barabasi, A.L.; Oltvai, Z.N. Network biology: Understanding the cell's functional organization. Nat. Rev. Genet. 2004, 5, 101-113. [CrossRef]

46. Milo, R.; Shen-Orr, S.; Itzkovitz, S.; Kashtan, N.; Chklovskii, D.; Alon, U. Network motifs: Simple building blocks of complex networks. Science 2002, 298, 824-827. [CrossRef]

47. Raman, K.; Damaraju, N.; Joshi, G.K. The organisational structure of protein networks: Revisiting the centrality-lethality hypothesis. Syst. Synth. Biol. 2014, 8, 73-81. [CrossRef]

48. Ashtiani, M.; Salehzadeh-Yazdi, A.; Razaghi-Moghadam, Z.; Hennig, H.; Wolkenhauer, O.; Mirzaie, M.; Jafari, M. A systematic survey of centrality measures for protein-protein interaction networks. BMC Syst. Biol. 2018, 12, 80. [CrossRef]

49. DeGeer, J.; Lamarche-Vane, N. Rho GTPases in neurodegeneration diseases. Exp. Cell Res. 2013, 319, 2384-2394. [CrossRef]

50. Tourette, C.; Li, B.; Bell, R.; O’Hare, S.; Kaltenbach, L.S.; Mooney, S.D.; Hughes, R.E. A large scale Huntingtin protein interaction network implicates Rho GTPase signaling pathways in Huntington disease. J. Biol. Chem. 2014, 289, 6709-6726. [CrossRef]

51. Shirasaki, D.I.; Greiner, E.R.; Al-Ramahi, I.; Gray, M.; Boontheung, P.; Geschwind, D.H.; Botas, J.; Coppola, G.; Horvath, S.; Loo, J.A.; et al. Network organization of the huntingtin proteomic interactome in mammalian brain. Neuron 2012, 75, 41-57. [CrossRef] [PubMed] 
52. Yao, Y.; Cui, X.; Al-Ramahi, I.; Sun, X.; Li, B.; Hou, J.; Difiglia, M.; Palacino, J.; Wu, Z.Y.; Ma, L.; et al. A striatal-enriched intronic GPCR modulates huntingtin levels and toxicity. eLife 2015, 4, e05449. [CrossRef] [PubMed]

53. Bae, B.I.; Xu, H.; Igarashi, S.; Fujimuro, M.; Agrawal, N.; Taya, Y.; Hayward, S.D.; Moran, T.H.; Montell, C.; Ross, C.A.; et al. p53 mediates cellular dysfunction and behavioral abnormalities in Huntington's disease. Neuron 2005, 47, 29-41. [CrossRef]

54. Krauss, S.; Griesche, N.; Jastrzebska, E.; Chen, C.; Rutschow, D.; Achmuller, C.; Dorn, S.; Boesch, S.M.; Lalowski, M.; Wanker, E.; et al. Translation of HTT mRNA with expanded CAG repeats is regulated by the MID1-PP2A protein complex. Nat. Commun. 2013, 4, 1511. [CrossRef] [PubMed]

55. Deyts, C.; Galan-Rodriguez, B.; Martin, E.; Bouveyron, N.; Roze, E.; Charvin, D.; Caboche, J.; Betuing, S. Dopamine D2 receptor stimulation potentiates PolyQ-Huntingtin-induced mouse striatal neuron dysfunctions via Rho/ROCK-II activation. PLoS ONE 2009, 4, e8287. [CrossRef]

56. Roscic, A.; Baldo, B.; Crochemore, C.; Marcellin, D.; Paganetti, P. Induction of autophagy with catalytic mTOR inhibitors reduces huntingtin aggregates in a neuronal cell model. J. Neurochem. 2011, 119, 398-407. [CrossRef]

57. Hong, H.; Koon, A.C.; Chen, Z.S.; Wei, Y.; An, Y.; Li, W.; Lau, M.H.Y.; Lau, K.F.; Ngo, J.C.K.; Wong, C.H.; et al. AQAMAN, a bisamidine-based inhibitor of toxic protein inclusions in neurons, ameliorates cytotoxicity in polyglutamine disease models. J. Biol. Chem. 2019, 294, 2757-2770. [CrossRef]

(C) 2020 by the authors. Licensee MDPI, Basel, Switzerland. This article is an open access article distributed under the terms and conditions of the Creative Commons Attribution (CC BY) license (http://creativecommons.org/licenses/by/4.0/). 